Anthony Sinskey

ORCID iD: 0000-0001-9433-4324

\title{
Optimizing Recombineering in Corynebacterium glutamicum
}

Cheng $\mathrm{Li}^{\mathrm{a}, \mathrm{b} *}$, Charles Swofford ${ }^{\mathrm{a}, \mathrm{b} *}$, Christian Rückert ${ }^{\mathrm{a}, \mathrm{b}}$, Anthony J. Sinskey ${ }^{\mathrm{a}, \mathrm{b} * *}$

${ }^{\mathrm{a}}$ Department of Biology, Massachusetts Institute of Technology, Cambridge, MA,

02139

${ }^{\mathrm{b}}$ Disruptive \& Sustainable Technologies for Agricultural Precision, Singapore-MIT Alliance for Research and Technology, Singapore, Singapore, 138602

*Authors contributed equally to this work

**Correspondence to:

Anthony J. Sinskey

Department of Biology

Massachusetts Institute of Technology

77 Massachusetts Ave., Bldg. 68

Cambridge, MA 02139-4301

Phone: (617) 253-6721

Fax: (617) 253-8550

Email: asinskey@mit.edu

Funding support: This work was supported by the Singapore-MIT Alliance for Research and Technology (SMART) program entitled Disruptive \& Sustainable Technologies for Agricultural Precision (DiSTAP).

\begin{abstract}
Due to increasing demand for amino acids and valuable commodities that can be produced by Corynebacterium glutamicum, there is a pressing need for new rapid genome engineering tools that improve the speed and efficiency of genomic This is the author manuscript accepted for publication and undergone full peer review but has not been through the copyediting, typesetting, pagination and proofreading process, which may lead to differences between this version and the Version of Record. Please cite this article as doi: 10.1002/bit.27737.
\end{abstract}

This article is protected by copyright. All rights reserved. 
insertions, deletions, and mutations. Recombineering using the $\lambda$ Red system in Escherichia coli has proven very successful at genetically modifying this organism in a quick and efficient manner, suggesting that optimizing a recombineering system for $C$. glutamicum will also improve the speed for genomic modifications. Here, we maximized recombineering efficiency in $C$. glutamicum by testing the efficacy of seven different recombinase/exonuclease pairs for integrating ssDNA and dsDNA into the genome. By optimizing the homologous arm length and the amount of dsDNA transformed, as well as eliminating codon bias, a dsDNA recombineering efficiency of 13,250 transformed colonies $/ 10^{9}$ viable cells was achieved, the highest efficiency currently reported in the literature. Using this optimized system, over 40,000 bp could be deleted in one transformation step. This recombineering strategy will greatly improve the speed of genetic modifications in C. glutamicum and assist other systems such as CRISPR and Multiplexed Automated Genome Engineering (MAGE) in improving targeted genome editing.

Keywords: Corynebacterium, recombineering, RecET, metabolic engineering, genomic engineering.

\section{Introduction}

Over the past 60 years, Corynebacterium glutamicum has been well known as an excellent producer of L-amino acids (Becker \& Wittmann, 2012). Recent advances in metabolic and synthetic biology have expanded the portfolio of chemicals that can be produced from C. glutamicum, but it is still difficult to This article is protected by copyright. All rights reserved. 
synthesize these compounds at an industrially relevant scale (S. Y. Kim et al., 2015; C. Li et al., 2020; L. Liu et al., 2013; Otten et al., 2015; Plassmeier et al., 2016; Woo \& Park, 2014; Xu et al., 2019; Zha et al., 2018). Due to the increasing worldwide demand of amino acids and other novel compounds, there is a pressing need for rapid improvement in strain engineering and production yields of chemical compounds in C. glutamicum (Wendisch, 2007). In order to construct novel metabolic pathways and redirect carbon flux, new tools are required that are optimized for C. glutamicum and improve the speed and efficiency of scarless genomic deletions, insertions, and mutations (Plassmeier et al., 2016; Woo \& Park, 2014).

The traditional approaches for strain engineering and genome modification in C. glutamicum are notoriously slow. Gene clusters can be inserted or deleted by a double-crossover homologous recombination event using a plasmid containing positive and negative selection markers, such as an antibiotic resistance cassette and the sacB gene (Schäfer et al., 1994). SacB can hydrolyze sucrose and synthesize levan, leading to sucrose sensitivity (Jäger et al., 1992). This process can take up to a week if successful and sequential deletions will only increase this time substantially. Another gene-editing method developed in C. glutamicum is based on the Cre/loxP system (Hu et al., 2013; Suzuki et al., 2007; Suzuki et al., 2005a; Suzuki et al., 2005b; Suzuki et al., 2005c; Suzuki et al., 2005d). Cre catalyzes reciprocal site-specific recombination between two loxP sites, leaving one loxP site (Gopaul et al., 1998) or a mutant loxLR site (Albert et al., 1995) on This article is protected by copyright. All rights reserved. 
the chromosome. Due to the low efficiency of homologous recombination in $C$. glutamicum, this method also proves slow and tedious. In addition, it is still constrained by the requirement for complex genetic constructions and leaves unwanted loxP scar sites in the genome.

Genetic engineering in Escherichia coli through recombineering using the $\lambda$ Red system or the recE and $\operatorname{rec} T$ genes of the Rac prophage has greatly improved genetic manipulation in a simple and efficient manner (Court et al., 2002; Zhang et al., 1998). These high recombination efficiencies can be exploited in various ways, including the construction of chromosomal gene knockouts, point mutations, deletions, small insertions, in vivo cloning, mutagenesis of bacterial artificial chromosomes and genomic libraries (Copeland et al., 2001; Court et al., 2002; Ellis et al., 2001; Sarov et al., 2006; Harris H Wang et al., 2009; H. H. Wang et al., 2012; Yu et al., 2000; Zhang et al., 2000). $\lambda$ Exo and RecE are 5'-3' double-stranded DNA (dsDNA) dependent exonucleases (Joseph \& Kolodner, 1983); $\lambda$ Beta and RecT are single-stranded DNA (ssDNA) annealing proteins (SSAPs) that promote annealing of complementary DNA strands, strand exchange and strand invasion (Z. Li et al., 1998; Noirot \& Kolodner, 1998). DsDNA recombination requires both an exonuclease and recombinase, whereas recombination using ssDNA substrates requires only the recombinase (Court et al., 2002). Five different recombinase-exonuclease pairs, Beta+Exo (Phage $\lambda$ of $E$. coli), GP35+GP34.1 (Bacillus subtilis), Orf48+Orf47 (Listeria monocytogenes), OrfC+OrfB (Legionella pneumophila), and plu2935+plu2936 (Photorhabdus This article is protected by copyright. All rights reserved. 
luminescens), have been tested for dsDNA recombination activity in E.coli, with Beta and Exo having the highest recombination efficiency (Datta et al., 2008). The $\lambda$ Beta and recT work less effectively, however, in Mycobacterium smegmatis (Van Kessel \& Hatfull, 2008), a close relative of C. glutamicum (Woese, 1987). GP60 and GP61 from mycobacteriophage Che9c, the RecE and RecT homologs, substantially elevates recombination facilitating allelic exchange in both $M$. smegmatis and Mycobacterium tuberculosis (Van Kessel \& Hatfull, 2007).

In C. glutamicum 13032, five different recombinases have been tested, with RecT having the highest recombineering efficiency (Binder et al., 2013). Recent studies have used this recombinase in C. glutamicum to develop new CRISPR-assisted genome editing (Cho et al., 2017; Jiang et al., 2017; H. J. Kim et al., 2020; J. Liu et al., 2017; Su et al., 2018; B. Wang et al., 2018), improve editing using the Cre/loxP system (Huang et al., 2017), and improve double-crossover homologous recombination using upp instead of $s a c B$ as a positive selection marker (Ma et al., 2015). DsDNA recombination in $C$. glutamicum using the RecET system, however, is still considered inefficient, and improvement in this system or other related recombineering systems need to be further explored (Jiang et al., 2017). Codon-optimization of these systems for $C$. glutamicum, for example, could improve efficiencies by increasing translation of the recombinase and exonuclease genes (Datta et al., 2008). Achieving higher efficiencies in recombineering will improve new CRISPR technologies being designed for $C$. glutamicum and understanding the advantages and limitations of This article is protected by copyright. All rights reserved. 
these recombineering systems will enable the use of other rapid genome engineering technologies, such as Multiplexed Automated Genome Engineering (MAGE), that are currently only optimized for a select few laboratory model strains (Nyerges et al., 2016).

In order to determine the optimal parameters for a recombineering system in C. glutamicum, we tested dsDNA recombination activity of recombinase/exonuclease pairs from seven sources: $B$. subtilis, phage $\lambda$ of $E$. coli, Rac prophage of E. coli, L. pneumophila, L. monocytogenes, P. luminescens, and phage Che9c of $M$. smegmatis. To optimize the recombination efficiency, we tested the effect of (i) different sizes of homology on either side of the DNA oligos targeting the genome, (ii) the amount of dsDNA needed for transformation, (iii) and the efficiency in a strain that eliminates codon-bias. For each recombinase/exonuclease pair, we tested both ssDNA oligos for point mutations and dsDNA for larger knockouts and insertions. Finally, we determined the maximum length of chromosome that could be knocked out under optimal conditions, as well as how many transformation cycles is required to knock out two or more distinct genomic loci. With these optimal parameters, we achieved the highest dsDNA recombineering efficiencies in C. glutamicum reported in the current literature.

This article is protected by copyright. All rights reserved. 


\section{Materials and methods}

\subsection{Strains and growth conditions}

\section{Bacterial strains and plasmids}

The present work involved the construction of different strains based on $C$. glutamicum ATCC 13032 (American Type Culture Collection). E. coli TOP10 F' (Invitrogen, US) was used for DNA manipulation. The E. coli-C. glutamicum shuttle expression vector pEC-XC99E (Kirchner \& Tauch, 2003) was used for introducing recombinase and exonuclease genes into C. glutamicum. All strains and plasmids used in this work are listed in Supplementary Table 1.

\section{Medium and cultivation}

E. coli TOP10 F' cells were cultivated in lysogeny broth (LB; Difco) medium at $37^{\circ} \mathrm{C}$. Bacterial plasmid selection and maintenance in E. coli was performed using $50 \mathrm{mg} / \mathrm{L}$ kanamycin (Invitrogen), $10 \mathrm{mg} / \mathrm{L}$ chloramphenicol (Invitrogen) or 25 mg/L tetracycline (Invitrogen). C. glutamicum derived strains were grown in BHI (37 g/L brain heart infusion (BD Difco)) medium. Recombinants were selected by BHI plates containing $7.5 \mathrm{mg} / \mathrm{L}$ chloramphenicol, $25 \mathrm{mg} / \mathrm{L}$ streptomycin (Invitrogen, US), $7.5 \mathrm{mg} / \mathrm{L}$ tetracycline or $25 \mathrm{mg} / \mathrm{L}$ kanamycin where necessary. BHIS (BHI supplemented with $91 \mathrm{~g} / \mathrm{L}$ sorbitol (Millipore-Sigma)) medium was prepared for making competent cells according to the descriptions of Van der Rest et al (Van der Rest et al., 1999). SOC outgrowth medium (BioLabs) was used for growing E. coli Top10 F' after transformation with plasmid DNA.

\subsection{Construction of vectors}

All molecular manipulations including PCR amplification, purification, digestion, This article is protected by copyright. All rights reserved. 
ligation, and electroporation were performed according to protocols described by Russell and Sambrook (Sambrook \& Russell, 2001). OneTaq Hot Start DNA Polymerase (New England Biolabs) was used for analytical purposes, while PCR products intended for sequencing and cloning were generated using Phusion DNA polymerase (New England Biolabs). Codon-optimized recE and recT for $C$. glutamicum were synthesized form Thermo Fisher Scientific. All primers used are listed in Supplementary Table 1.

\subsection{Construction of C. glutamicum recombinant strains}

The recombinase and exonuclease genes were cloned into the constitutive expression vector pEC-XC99E, a plasmid containing both a C. glutamicum and E. coli origin of replication as well as a chloramphenicol resistance cassette. This plasmid was constructed through Gibson assembly and the NEBuilder HiFi DNA Assembly Master Mix (New England Biolabs), using the primers as described in Supplementary Table 1. Preparation of the competent C. glutamicum cells as well as electroporation was carried out by the method of Dunican and Shivnan (Dunican \& Shivnan, 1989) and Van der Rest et al (Van der Rest et al., 1999). The plasmid was transferred into C. glutamicum cells by electroporation, and the recombinant strains were screened on BHI plates containing chloramphenicol. The details of constructing the codon-unbiased strain are provided in the Supplementary methods.

\subsection{Recombineering protocol in C. glutamicum}

All the dsDNA fragments were amplified by PCR or fusion PCR with the primers

This article is protected by copyright. All rights reserved. 
given in Supplementary Table 1.

For C. glutamicum recombineering, preparation of the competent $C$. glutamicum cells as well as electroporation was carried out by the protocol laid out in Stephan Binder et al. (Binder et al., 2013) with modification. Strains carrying either an empty vector, a vector expressing a recombinase or a vector expressing a recombinase and exonuclease pair were inoculated in fresh BHI medium contained $7.5 \mathrm{mg} / \mathrm{L}$ chloramphenicol (BHIC) for 16 hours. This pre-culture was innoculated into $50 \mathrm{~mL}$ BHIC medium contained $0.1 \%$ tween 80 , $2 \%$ glycine and $2 \mathrm{mM}$ isopropyl- $\beta$-d-thiogalactoside (IPTG, Invitrogen), at an $\mathrm{OD}_{600}$ of 0.2 . Four hours later, OD was measured and $10^{9}$ cells were harvested and made electrocompetent. They were chilled on ice for $15 \mathrm{~min}$ and then harvested at 4,000 rpm and $4{ }^{\circ} \mathrm{C}$ for $5 \mathrm{~min}$, washed once in $50 \mathrm{~mL}$ of TG-buffer ( $1 \mathrm{mM}$ Tris$\mathrm{HCl}, \mathrm{pH}$, and $10 \%$ glycerol) and once in $50 \mathrm{~mL}$ of $10 \%$ glycerol. The competent cells were then resuspended in $1 \mathrm{~mL}$ of $10 \%$ glycerol and used immediately. The competent cells were thawed on ice and mixed with ssDNA or dsDNA fragment and transferred into $4{ }^{\circ} \mathrm{C}$ pre-cooled electroporation cuvettes. Electroporation was performed at $25 \mu \mathrm{F}, 200 \Omega$ and $2.5 \mathrm{kV}$. Immediately afterwards, cells were transferred into $4 \mathrm{~mL}$ of pre-warmed BHIS medium containing $7.5 \mathrm{mg} / \mathrm{L}$ chloramphenicol and heat shocked for 6 min at $46{ }^{\circ} \mathrm{C}$. They then recovered for $5 \mathrm{~h}$ at $30{ }^{\circ} \mathrm{C}$ and $200 \mathrm{rpm}$. Cells were plated on BHI plates with appropriate antibiotics at $30{ }^{\circ} \mathrm{C}$ for 2 days. Colonies were counted on the plates after this time. In addition to the ssDNA or dsDNA, one aliquot was used as a This article is protected by copyright. All rights reserved. 
control by transforming with 500 ng of plasmid DNA, either pZ8-rpsL ${ }^{\text {mutatedA128G }}$ (Supplementary Table 1) conferring streptomycin resistance or pZ8-1 (Supplementary Table 1) conferring kanamycin resistance.

For multiplexed genome engineering, after electroporation and five hours of recovery, cells were cultured at $30{ }^{\circ} \mathrm{C}$ and $20 \mathrm{rpm}$ for an additional 12 hours. They were then used to prepare competent cells for transformation with dsDNA fragments and recovered as described above. This process is considered one cycle and was repeated for additional cycles as indicated.

\section{Results}

\subsection{Generating point-mutations using ssDNA recombineering in $C$.}

\section{glutamicum}

Seven recombinases were tested in C. glutamicum ATCC 13032 to determine their functionality: Beta (Phage $\lambda$ of E. coli), GP35 (B. subtilis), Orf48 (L. monocytogenes), OrfC (L. pneumophila), plu2935 (P. luminescens), RecT (Rac prophage of E. coli) (Datta et al., 2008), and GP61 (Phage Che9c of $M$. smegmatis). Unregulated expression of recombinases can be toxic to bacterial hosts (Court et al., 2002; Sergueev et al., 2001), so each recombinase was cloned into the IPTG-inducible expression vector, pEC-XC99E (Kirchner \& Tauch, 2003). In order to evaluate recombination efficiency of each recombinase, the ssDNA oligo rpsL ${ }^{\text {mutatedA128G }}$ was used to target an A to $\mathrm{G}$ transversion in $r p s L$ that confers streptomycin resistance $\left(\mathrm{Str}^{\mathrm{R}}\right)$ (Böttger et al., 2001).

This article is protected by copyright. All rights reserved. 
Three out of the seven recombinases tested showed a significant increase in recombination efficiency compared to controls that did not receive oligos: RecT, GP61, and OrfC (Table 1). RecT had the highest recombineering efficiency in $C$. glutamicum, with $\sim 4800 \mathrm{Str}^{\mathrm{R}}$ colonies per $10^{9}$ cells, an order of magnitude higher than GP61 or OrfC. Sequencing confirmed that cells targeted using these three recombinases conferred the correct A128G point mutation in $90 \%$ of cases, while the $\operatorname{Str}^{\mathrm{R}}$ colonies targeted using the remaining four recombinases showed random mutations in the rpsL gene. These results confirm similar results by Binder et. al. (Binder et al., 2013).

\subsection{Whole genome insertions using dsDNA recombineering in C. glutamicum}

While small single point mutations are important for modifying the genome, the majority of strain engineering will involve large scale genomic insertions and deletions. This requires not only a recombinase, but also a complementary exonuclease that is required to recombine heterologous dsDNA. In order to test genome insertion efficiency, a kanamycin-resistance $\left(\mathrm{Km}^{\mathrm{R}}\right)$ cassette was used in conjunction with seven different recombinase-exonuclease pairs to replace the nonessential gene cgp_2810 (Figure 1), which encodes for the glutamate/dicarboxylate symporter. This cassette contained 400 bp homologous arms flanking the kanamycin-resistance gene. All recombinase-exonuclease pairs were cloned into the IPTG-inducible plasmid pEC-XC99E.

Similar to the results with ssDNA, three recombinase-exonuclease pairs exhibited functionality in C. glutamicum and conferred kanamycin resistance: This article is protected by copyright. All rights reserved. 
GP61/GP60, OrfC/OrfB, and RecT/RecE (Figure 2A). The RecT/RecE pair had the highest recombineering efficiency with over $1200 \mathrm{Km}^{\mathrm{R}}$ cells per $10^{9}$ cells, an order of magnitude higher than GP61/GP60. As expected, using only the recombinase when transforming heterologous dsDNA yielded no colonies in all cases (Supplementary Table 2). No $\mathrm{Km}^{\mathrm{R}}$ colonies were formed using any other recombinase/exonuclease pair or with negative controls.

\subsection{Optimizing dsDNA Recombineering in C. glutamicum}

The RecT/E recombinase/exonuclease pair demonstrated the highest efficiency in C. glutamicum for both point mutations and large genome insertions, but other parameters such as homologous arm length and dsDNA amount must be optimized to determine the highest achievable efficiency. A kanamycin-resistance cassette flanked by homologous arms ranging from 20 bp to 2,000 bp was generated by PCR or fusion PCR and transformed using 500 ng of dsDNA to replace the nonessential gene cgp_2810. $25 \mathrm{Km}^{\mathrm{R}}$ cells per $10^{9}$ viable cells were obtained when the homologous arm length was 50 bp, with increasing efficiency at larger arm lengths (Figure 2B). A maximum efficiency of 5,500 $\mathrm{Km}^{\mathrm{R}}$ cells per $10^{9}$ viable cells was achieved with a homologous arm length of $1,000 \mathrm{bp}$, with decreasing efficiency at larger arm lengths (Figure 2B). This resulted in a 5-fold increase in efficiency compared to the 400 bp homologous arm lengths used in earlier experiments (Figure 2B).

With the optimal homologous arm length of 1,000 bp, we next determined the highest efficiency based on the amount of dsDNA transformed. Maximum This article is protected by copyright. All rights reserved. 
transformation efficiency of $6700 \mathrm{Km}^{\mathrm{R}}$ cells per $10^{9}$ viable cells was achieved when using $1 \mu \mathrm{g}$ of dsDNA, although higher amounts did not affect the transformation efficiency greatly (Figure 2C). As the amount of dsDNA increased, however, the amount of $\mathrm{Km}^{\mathrm{R}}$ cells per $\mu \mathrm{g}$ of DNA decreased, indicating that dsDNA amounts higher than $1 \mu \mathrm{g}$ are unnecessary and inefficient.

Finally, the optimal parameters for recombineering were used to determine the largest length of the genome that could be deleted in one recombination event. Using RecE/T, $1 \mu \mathrm{g}$ of dsDNA, and 1,000 bp homologous arm lengths, genomic regions ranging from 1,000 bp to 40,000 bp were targeted for deletion between the regions of cgp_0702 and cgp_0750 (Figure 2D), which is required for carotenoid biosynthesis (S. A. Heider et al., 2012). This region was determined to be unessential due to similar growth rates with the WT upon deletion (Supplementary Figure 1). All deletions removed cgp_0721 that encodes for phytoene synthase, which in turn prevents lycopene synthesis and renders the typically yellow Corynebacterium colonies white (S. A. Heider et al., 2012), allowing for an easy method of screening. With these optimal parameters, RecE/T recombination could delete a 40,000 bp region in the genome at an efficiency of 2,600 knockouts/10 viable cells. The highest efficiency of 6,217 knockouts $/ 10^{9}$ viable cells was achieved when deleting a 10,000 bp region. Efficiency decreased substantially when deleting higher lengths of the genome.

This article is protected by copyright. All rights reserved. 


\subsection{Codon-Unbiased Corynebacterium Strain Improves Recombination}

\section{Efficiency}

In order to alleviate any codon bias between different recombinant proteins from different organisms, each recombinase/exonuclease pair was tested in a codon-unbiased strain (cg203). Cg203 was engineered to overexpress 28 rare codon tRNAs by inserting a tRNA ${ }^{\text {Leu }}$ GAG promoter. This eliminates codon-bias effects in heterologous genes that are not optimized for C. glutamicum. Each test was done with 500 ng dsDNA and 400 bp homolgous arms to directly compare to the initial results (Table 1 and Figure 2A). First, we once again tested ssDNA recombineering using the ssDNA oligo $\mathrm{rps}^{\text {mutatedA128G }}$ to confer streptomycin resistance and determined that efficiency was improved in all cases (Figure 3A; $\mathrm{P}<0.05)$. A maximum transformation efficiency of $7262 \mathrm{Str}^{\mathrm{R}}$ colonies per $10^{9}$ viable cells was achieved with the RecT recombinase when used with the codon-optimized cg203 strain. This is a 1.5 fold higher efficiency than the same test conducted in the wild-type strain. Many other recombinases exhibited higher fold increases in cg203 compared to the WT. Orf48, for example, showed an increase from 24 to $122 \mathrm{Str}^{\mathrm{R}}$ colonies per $10^{9}$ viable cells when used in cg203 compared to the WT, a 4.7 fold increase in recombination efficiency.

In addition, dsDNA recombineering was tested in the codon-optimized strain cg203 by once again inserting a kanamycin-resistant cassette into the nonessential gene cgp_2810. All recombinase/exonuclease pairs except Bet/Exo and GP35/34.1 yielded $\mathrm{Km}^{\mathrm{R}}$ clones in cg203 and improved their efficiency as This article is protected by copyright. All rights reserved. 
compared to the wild-type (Figure 3B, $\mathrm{P}<0.05$ ). Two pairs, Orf48/47 and plu2935/2936, yielded $\mathrm{Km}^{\mathrm{R}}$ colonies when used in the cg203 strain that previously showed no recombinatorial effect in the wild-type strain. Once again, RecE/T demonstrated the highest efficiency with $1853 \mathrm{Km}^{\mathrm{R}}$ colonies per $10^{9}$ viable cells and a 1.6 fold increase in efficiency compared to using the pair in the wild-type strain. A codon-optimized recE/T was synthesized and transformed into the wild-type strain, which gave similar results compared to the non codon-optimized recE/T in cg203 (Supplementary Figure 2). As a final test, optimized conditions ( $1 \mu \mathrm{g}$ and 1,000 bp homologous arms) were used with RecET in cg203 to determine the highest efficiency achievable with the kanamycin-resistance cassette. Optimal conditions with cg203 yielded an efficiency of $9800 \mathrm{Km}^{\mathrm{R}}$ colonies per $10^{9}$ viable cells, 1.4 fold higher than in the wild-type (Figure 4A).

\subsection{The RecET System Allows for Two and Three Separate Genomic}

\section{Insertions}

To test the recombineering efficiency of different location sites, cgp_2588 that encodes for glutamate 5-kinase and cgp_0717 that encodes for lycopene elongase, both of which are non-essential genes in C. glutamicum (S. A. Heider et al., 2012), were chosen as well as cgp_0721. Meanwhile, both a kanamycin-resistance cassette and tetracycline-resistance $\left(\right.$ Tet $^{\mathrm{R}}$ ) cassette were tested (Figure 4A). Different location sites had varying recombineering efficiencies in strain cg203, with cgp_2588 site obtaining the lowest efficiency of $9200 \mathrm{Km}^{\mathrm{R}}$ colonies $/ 10^{9}$ This article is protected by copyright. All rights reserved. 
viable cells while cgp_0717 obtaining the highest efficiency of $13250 \mathrm{Km}^{\mathrm{R}}$ colonies $/ 10^{9}$ viable cells. The insertion of the kanamycin-resistance cassette or the tetracycline-resistance cassette had similar recombineering efficiencies at the same site (Figure 4A).

As a final test of the system, the RecET pair was used under optimal conditions (1 $\mu$ g DNA and 1,000 bp homolgous arms) to determine if two and three distinct cassettes could be transformed into C. glutamicum with one transformation event. In order to run this experiment, three fragments were used, including the previously described kanamycin-resistance cassette to remove the nonessential region cgp_0702-cgp_0750, a tetracycline-resistance cassette designed to integrate cgp 0720, cgp 0721 and cgp 0723 that encode for phytoene desaturase, phytoene synthase and geranylgeranyl pyrophosphate synthase to produce lycopene and make the colony red (Supplementary Figure 3) (S. A. Heider et al., 2012), and a fragment carrying the $r p s L^{\text {mutatedA128G }}$ mutation to obtain streptomycin resistance. One, two or three of these fragments were transformed into both the wild-type and cg203 strain. Even though the efficiency was greatly reduced compared to using a single cassette, the RecET system could recombine two distinct cassettes at an efficiency of $7 \mathrm{Str}^{\mathrm{R}}+\mathrm{Km}^{\mathrm{R}}$ colonies, $6 \mathrm{Km}^{\mathrm{R}}+\mathrm{Tet}^{\mathrm{R}}$ colonies and $10 \mathrm{Str}^{\mathrm{R}}+\mathrm{Km}^{\mathrm{R}}$ per $10^{9}$ viable cells in the wild-type strain (Figure 4B). This efficiency was improved 1.7 fold, 1.7 fold and 1.6 fold when using the codon-optimized strain, cg203. Attempts using three distinct cassettes each containing a different antibiotic This article is protected by copyright. All rights reserved. 
marker yielded no colonies (Figure 4B), suggesting that the system can only recombine two distinct cassettes at maximum with one transformation step.

To increase the recombineering efficiency, we multiplexed the transformation. After transformation and the recovery phase, cells were made electrocompetent for the next step of transformation and recovery. This was considered one cycle. The recombinants carrying one or two resistance cassettes increased with increasing cycles (Supplementary Figure 4). After 4 cycles, an efficiency of 2 $\mathrm{Str}^{\mathrm{R}}+\mathrm{Km}^{\mathrm{R}}+\mathrm{Tet} \mathrm{R}^{\mathrm{R}}$ colonies per $10^{9}$ viable cells was achieved in the wild-type strain (Figure 4C). This efficiency was improved 4-fold when using the codon-optimized strain, cg203.

\section{Discussion}

Recombineering in C. glutamicum is most effective and efficient when the RecET system from the Rac prophage of $E$. coli is used. We have tested seven different recombinase/exonuclease pairs from various organisms and have reported the highest efficiencies in the literature for dsDNA recombineering. This was achieved by optimizing the amount of dsDNA template and the length of the flanking homologous regions. These optimal parameters allow for genomic deletions of upwards of 40,000 bp in one recombineering event. Efficiencies of each recombinase/exonuclease pair was improved further by recombineering in a codon-unbiased strain. Finally, RecET recombineering in C. glutamicum can insert two separate genes into distinct separate genomic locations with one recombineering event and insert three separate genes with four recombineering This article is protected by copyright. All rights reserved. 
cycles. This optimized system will be a useful tool for rapid metabolic engineering C. glutamicum.

Previous research using RecET in Brevibacterium sp. DSM 20411 (Huang et al., 2017), which was renamed recently from Corynebacterium glutamicum ATCC 14067 and is considered to be related to C. glutamicum ATCC 13032 (Lv et al., 2012), reached efficiencies of 1,410 colonies/mL under optimized conditions, still considered rather low when compared to efficiencies in E. coli that are 1,000 fold higher (Nakashima \& Miyazaki, 2014). Under the optimal conditions outlined here, RecET in C. glutamicum ATCC 13032 achieved efficiencies as high as 3,312 colonies/mL, 2.35 fold higher than previously reported (Huang et al., 2017) (Supplementary table 3). This is the highest reported dsDNA recombination efficiency reported for C. glutamicum currently in the literature. This increase can be attributed to using a codon-unbiased strain, as well as the optimal homology length of 1,000 bp and dsDNA amount of $1 \mu g$ that was identified. In addition, colonies were obtained using a homology length of $50 \mathrm{bp}$, allowing a user to use synthesized oligos and save the effort of using fusion PCR to create longer arms. Other recombination systems, including three (GP61/60, GP35/34.1, and plu2935/2936) that have never been tested in C. glutamicum, were compared to RecET in the hope that a more efficient system could be discovered, but RecET consistently performed the best. All the systems improved their efficiencies with the codon-unbiased strain for both dsDNA and ssDNA recombineering but surprisingly, two recombinase/exonuclease pairs, Orf48/47 and plu2935/2936, This article is protected by copyright. All rights reserved. 
only worked for dsDNA recombineering in the codon unbiased strain. While the efficiencies remained low, this illustrates the importance of codon optimization when using heterologous genes to maximize expression and improving function.

Ultimately, the goal for genomic editing would be to engineer a system that can edit any location in the genome in a short amount of time, leave no genomic scars, and provide a simple screening method. The system outlined here can meet two out of the three criteria: it can either leave an antibiotic resistance marker as a scar or it can leave a scarless edit without the ability to screen for positive mutants. Previous work combining RecET recombineering with the Cre/loxP system created a self-excisable cassette to improve genome editing, but this system still leaves a lox72 site in the genome where editing took place (Huang et al., 2017; Suzuki et al., 2005d). Combining the optimized RecET recombineering system with CRISPR-Cas9 (B. Wang et al., 2018) or CRISPR-Cpf1 (Zhao et al., 2020) can overcome these challenges by leaving scarless edits, but these tools are still limited by off-target effects and the inability to delete large genomic regions (Jiang et al., 2017; Wu et al., 2020). RecET recombineering allows for any length of the genome to be deleted to upwards of 40,000 bp with higher lengths possible (Figure 2D). Recent work with nickase versions of Cas9 in E.coli to delete large genomic regions suggest that creating a nickase version of Cpf1 might improve the editing efficiency in C. glutamicum as well (Standage-Beier et al., 2015; Zhao et al., 2020). Combining these new CRISPR-based methods with the optimal parameters outlined here for RecET recombineering has the potential to provide This article is protected by copyright. All rights reserved. 
higher efficiencies and allow for the freedom to choose any location and length in the genome to edit (Ronda et al., 2016; Zhu et al., 2017).

Perhaps the greatest technology to benefit from improved recombineering in $C$. glutamicum is multiplexed automated genome engineering (MAGE). MAGE has been developed for E. coli using the lambda red recombineering machinery to enable the rapid and continuous generation of sequence diversity at many targeted chromosomal locations across a large population of cells, but it has only been tried with ssDNA recombination to mutate RBS sites or insert nonsense mutations into genes (H. H. Wang et al., 2009). By using the exonuclease and recombinase during each MAGE cycle, dsDNA can be used to do large scale genomic deletions or insertions (Figure 2), thereby improving the genetic diversity of potential strains producing an intended target. Development of a MAGE system in $C$. glutamicum using the RecET system will eliminate the need for targeting each gene in a pathway sequentially, instead targeting an entire pathway at once. For each MAGE cycle, the RecET system has the potential to insert or delete two distinct regions in the chromosome (Figure 4B). Potential targets of interest, such as lycopene and its derivatives (S. A. E. Heider et al., 2014), can be easily screened for by the pigment change in the colony. More ambitious goals, such as developing a chassis genome for $C$. glutamicum, will be achievable sooner with RecET recombineering to delete unwanted gene clusters (Unthan et al., 2015). Overall, the optimal RecET recombineering system outlined here provides a 
necessary tool to advance genome engineering in C. glutamicum and improve the production of compounds of interest.

\section{Competing interests}

The authors declare that they have no competing interests.

\section{Authors' contributions}

C.L., C.A.S., and A.J.S. designed the research; C.L. performed the research; C.L. and C.A.S. analyzed data; and C.L. and C.A.S. wrote the paper.

\section{Acknowledgements}

We gratefully acknowledge financial support from the Singapore-MIT Alliance for Research and Technology (SMART) program entitled Disruptive \& Sustainable Technologies for Agricultural Precision (DiSTAP). We acknowledge supply of the plasmids carried recombinase/exonuclease pairs by Donald L. Court.

\section{References}

Albert, H., Dale, E. C., Lee, E., \& Ow, D. W. (1995). Site-specific integration of DNA into wildtype and mutant lox sites placed in the plant genome. The Plant Journal, 7(4), 649-659.

Becker, J., \& Wittmann, C. (2012). Bio-based production of chemicals, materials and fuelsCorynebacterium glutamicum as versatile cell factory. Current opinion in biotechnology, 23(4), 631-640.

Binder, S., Siedler, S., Marienhagen, J., Bott, M., \& Eggeling, L. (2013). Recombineering in Corynebacterium glutamicum combined with optical nanosensors: a general strategy for fast producer strain generation. Nucleic acids research, gkt312.

Böttger, E. C., Springer, B., Prammananan, T., Kidan, Y., \& Sander, P. (2001). Structural basis for selectivity and toxicity of ribosomal antibiotics. EMBO reports, 2(4), 318-323.

Cho, J. S., Choi, K. R., Prabowo, C. P. S., Shin, J. H., Yang, D., Jang, J., \& Lee, S. Y. J. M. e. (2017). CRISPR/Cas9-coupled recombineering for metabolic engineering of Corynebacterium glutamicum. 42, 157-167.

Copeland, N. G., Jenkins, N. A., \& Court, D. L. (2001). Recombineering: a powerful new tool for mouse functional genomics. Nature Reviews Genetics, 2(10), 769-779.

Court, D. L., Sawitzke, J. A., \& Thomason, L. C. (2002). Genetic Engineering Using Homologous Recombination 1. Annual review of genetics, 36(1), 361-388.

Datta, S., Costantino, N., \& Zhou, X. (2008). Identification and analysis of recombineering

This article is protected by copyright. All rights reserved. 
functions from Gram-negative and Gram-positive bacteria and their phages. Proceedings of the National Academy of Sciences, 105(5), 1626-1631.

Dunican, L. K., \& Shivnan, E. (1989). High frequency transformation of whole cells of amino acid producing coryneform bacteria using high voltage electroporation. Nature biotechnology, 7(10), 1067-1070.

Ellis, H. M., Yu, D., \& DiTizio, T. (2001). High efficiency mutagenesis, repair, and engineering of chromosomal DNA using single-stranded oligonucleotides. Proceedings of the National Academy of Sciences, 98(12), 6742-6746.

Gopaul, D. N., Guo, F., \& Van Duyne, G. D. (1998). Structure of the Holliday junction intermediate in Cre-loxP sitespecific recombination. The EMBO Journal, 17(14), 4175-4187.

Heider, S. A., Peters-Wendisch, P., \& Wendisch, V. F. J. B. m. (2012). Carotenoid biosynthesis and overproduction in Corynebacterium glutamicum. 12(1), 198.

Heider, S. A. E., Wolf, N., Hofemeier, A., Peters-Wendisch, P., \& Wendisch, V. F. (2014). Optimization of the IPP Precursor Supply for the Production of Lycopene, Decaprenoxanthin and Astaxanthin by Corynebacterium glutamicum. Frontiers in bioengineering and biotechnology, 2, 28-28. doi:10.3389/fbioe.2014.00028

Hu, J., Tan, Y., Li, Y., Hu, X., Xu, D., \& Wang, X. (2013). Construction and application of an efficient multiple-gene-deletion system in Corynebacterium glutamicum. Plasmid, 70(3), 303-313.

Huang, Y. Y., Li, L., Xie, S., Zhao, N. N., Han, S. Y., Lin, Y., \& Zheng, S. P. (2017). Recombineering using RecET in Corynebacterium glutamicum ATCC14067 via a self-excisable cassette. Scientific Reports, 7, 8. doi:10.1038/s41598-017-08352-9

Jäger, W., Schäfer, A., Pühler, A., Labes, G., \& Wohlleben, W. (1992). Expression of the Bacillus subtilis sacB gene leads to sucrose sensitivity in the gram-positive bacterium Corynebacterium glutamicum but not in Streptomyces lividans. Journal of bacteriology, 174(16), 5462-5465.

Jiang, Y., Qian, F. H., Yang, J. J., Liu, Y. M., Dong, F., Xu, C. M.,... Yang, S. (2017). CRISPR-Cpf1 assisted genome editing of Corynebacterium glutamicum. Nature Communications, 8, 11. doi:10.1038/ncomms15179

Joseph, J. W., \& Kolodner, R. (1983). Exonuclease VIII of Escherichia coli. II. Mechanism of action. Journal of Biological Chemistry, 258(17), 10418-10424.

Kim, H. J., Oh, S. Y., \& Lee, S. J. (2020). Efficient and Accurate Single-Base Genome Editing in Corynebacterium Glutamicum by Target-Mismatched CRISPR/Cpf1.

Kim, S. Y., Lee, J., \& Lee, S. Y. (2015). Metabolic engineering of Corynebacterium glutamicum for the production of L-ornithine. Biotechnology and bioengineering, 112(2), 416-421.

Kirchner, O., \& Tauch, A. (2003). Tools for genetic engineering in the amino acid-producing bacterium Corynebacterium glutamicum. Journal of biotechnology, 104(1), 287-299.

Li, C., Swofford, C. A., \& Sinskey, A. J. J. M. E. C. (2020). Modular engineering for microbial production of carotenoids. 10, e00118.

Li, Z., Karakousis, G., Chiu, S., Reddy, G., \& Radding, C. (1998). The beta protein of phage $\lambda$ promotes strand exchange. Journal of molecular biology, 276(4), 733-744.

Liu, J., Wang, Y., Lu, Y., Zheng, P., Sun, J., \& Ma, Y. J. M. c. f. (2017). Development of a CRISPR/Cas9 genome editing toolbox for Corynebacterium glutamicum. 16(1), 205.

This article is protected by copyright. All rights reserved. 
Liu, L., Yang, H., Shin, H.-d., Li, J., Du, G., \& Chen, J. (2013). Recent advances in recombinant protein expression by Corynebacterium, Brevibacterium, and Streptomyces: from transcription and translation regulation to secretion pathway selection. Applied Microbiology and Biotechnology, 97(22), 9597-9608. doi:10.1007/s00253-013-5250-x

Lv, Y., Liao, J., Wu, Z., Han, S., Lin, Y., \& Zheng, S. (2012). Genome sequence of Corynebacterium glutamicum ATCC 14067, which provides insight into amino acid biosynthesis in coryneform bacteria. In: Am Soc Microbiol.

Ma, W. W., Wang, X. Y., Mao, Y. F., Wang, Z. W., Chen, T., \& Zhao, X. M. (2015). Development of a markerless gene replacement system in Corynebacterium glutamicum using upp as a counter-selection marker. Biotechnology Letters, 37(3), 609-617. doi:10.1007/s10529-014-1718-8

Nakashima, N., \& Miyazaki, K. (2014). Bacterial Cellular Engineering by Genome Editing and Gene Silencing. International Journal of Molecular Sciences, 15(2), 2773-2793. doi:10.3390/ijms15022773

Noirot, P., \& Kolodner, R. D. (1998). DNA strand invasion promoted by Escherichia coli RecT protein. Journal of Biological Chemistry, 273(20), 12274-12280.

Nyerges, A., Csorgo, B., Nagy, I., Balint, B., Bihari, P., Lazar, V.,... Pal, C. (2016). A highly precise and portable genome engineering method allows comparison of mutational effects across bacterial species. Proceedings of the National Academy of Sciences of the United States of America, 113(9), 2502-2507. doi:10.1073/pnas.1520040113

Otten, A., Brocker, M., \& Bott, M. (2015). Metabolic engineering of Corynebacterium glutamicum for the production of itaconate. Metabolic engineering, 30, 156-165.

Plassmeier, J., Li, Y., Rueckert, C., \& Sinskey, A. J. (2016). Metabolic engineering Corynebacterium glutamicum to produce triacylglycerols. Metabolic engineering, 33, 86-97.

Ronda, C., Pedersen, L. E., Sommer, M. O., \& Nielsen, A. T. (2016). CRMAGE: CRISPR optimized mage recombineering. Scientific reports, 6(1), 1-11.

Sambrook, J., \& Russell, D. W. (2001). Molecular cloning: a laboratory manual 3rd edition. Coldspring-Harbour Laboratory Press, UK.

Sarov, M., Schneider, S., Pozniakovski, A., Roguev, A., Ernst, S., Zhang, Y.,... Stewart, A. F. (2006). A recombineering pipeline for functional genomics applied to Caenorhabditis elegans. Nature methods, 3(10), 839-844.

Schäfer, A., Tauch, A., Jäger, W., Kalinowski, J., Thierbach, G., \& Pühler, A. (1994). Small mobilizable multi-purpose cloning vectors derived from the Escherichia coli plasmids pK18 and pK19: selection of defined deletions in the chromosome of Corynebacterium glutamicum. Gene, 145(1), 69-73.

Sergueev, K., Yu, D., Austin, S., \& Court, D. (2001). Cell toxicity caused by products of the p L operon of bacteriophage lambda. Gene, 272(1), 227-235.

Standage-Beier, K., Zhang, Q., \& Wang, X. (2015). Targeted Large-Scale Deletion of Bacterial Genomes Using CRISPR-Nickases. Acs Synthetic Biology, 4(11), 1217-1225. doi:10.1021/acssynbio.5b00132

Su, T., Jin, H., Zheng, Y., Zhao, Q., Chang, Y., Wang, Q.,... Biotechnology. (2018). Improved ssDNA recombineering for rapid and efficient pathway engineering in Corynebacterium glutamicum. 93(12), 3535-3542.

This article is protected by copyright. All rights reserved. 
Suzuki, N., Inui, M., \& Yukawa, H. (2007). Site-directed integration system using a combination of mutant lox sites for Corynebacterium glutamicum. Applied microbiology and biotechnology, 77(4), 871-878.

Suzuki, N., Nonaka, H., Tsuge, Y., Inui, M., \& Yukawa, H. (2005a). New multiple-deletion method for the Corynebacterium glutamicum genome, using a mutant lox sequence. Applied and environmental microbiology, 71(12), 8472-8480.

Suzuki, N., Nonaka, H., Tsuge, Y., Okayama, S., Inui, M., \& Yukawa, H. (2005b). Multiple large segment deletion method for Corynebacterium glutamicum. Applied microbiology and biotechnology, 69(2), 151-161.

Suzuki, N., Okayama, S., Nonaka, H., Tsuge, Y., Inui, M., \& Yukawa, H. (2005c). Large-scale engineering of the Corynebacterium glutamicum genome. Applied and environmental microbiology, 71(6), 3369-3372.

Suzuki, N., Tsuge, Y., Inui, M., \& Yukawa, H. (2005d). Cre/loxP-mediated deletion system for large genome rearrangements in Corynebacterium glutamicum. Applied microbiology and biotechnology, 67(2), 225-233.

Unthan, S., Baumgart, M., Radek, A., Herbst, M., Siebert, D., Bruehl, N.,.. Noack, S. (2015). Chassis organism from Corynebacterium glutamicum - a top-down approach to identify and delete irrelevant gene clusters. Biotechnology Journal, 10(2), 290-301. doi:10.1002/biot.201400041

Van der Rest, M., Lange, C., \& Molenaar, D. (1999). A heat shock following electroporation induces highly efficient transformation of Corynebacterium glutamicum with xenogeneic plasmid DNA. Applied microbiology and biotechnology, 52(4), 541-545.

Van Kessel, J. C., \& Hatfull, G. F. (2007). Recombineering in Mycobacterium tuberculosis. Nature methods, 4(2), 147-152.

Van Kessel, J. C., \& Hatfull, G. F. (2008). Efficient point mutagenesis in mycobacteria using single-stranded DNA recombineering: characterization of antimycobacterial drug targets. Molecular microbiology, 67(5), 1094-1107.

Wang, B., Hu, Q., Zhang, Y., Shi, R., Chai, X., Liu, Z.,... Wen, T. J. M. c. f. (2018). A RecET-assisted CRISPR-Cas9 genome editing in Corynebacterium glutamicum. 17(1), 63.

Wang, H. H., Isaacs, F. J., Carr, P. A., Sun, Z. Z., Xu, G., Forest, C. R., \& Church, G. M. (2009). Programming cells by multiplex genome engineering and accelerated evolution. Nature, 460(7257), 894-U133. doi:10.1038/nature08187

Wang, H. H., Kim, H., Cong, L., Jeong, J., Bang, D., \& Church, G. M. (2012). Genome-scale promoter engineering by coselection MAGE. Nature methods, 9(6), 591-593.

Wendisch, V. F. (2007). Amino acid biosynthesis-pathways, regulation and metabolic engineering (Vol. 5): Springer Science \& Business Media.

Woese, C. R. (1987). BACTERIAL EVOLUTION. Microbiological Reviews, 51(2), 221-271.

Woo, H. M., \& Park, J.-B. (2014). Recent progress in development of synthetic biology platforms and metabolic engineering of Corynebacterium glutamicum. Journal of biotechnology, 180, 43-51.

Wu, Y., Liu, Y., Lv, X., Li, J., Du, G., \& Liu, L. (2020). Applications of CRISPR in a Microbial Cell Factory: From Genome Reconstruction to Metabolic Network Reprogramming. Acs Synthetic Biology, 9(9), 2228-2238. doi:10.1021/acssynbio.0c00349

This article is protected by copyright. All rights reserved. 
Xu, G., Zha, J., Cheng, H., Ibrahim, M. H., Yang, F., Dalton, H.,... Chi, K. (2019). Engineering Corynebacterium glutamicum for the de novo biosynthesis of tailored poly- $\gamma$-glutamic acid. Metabolic engineering, 56, 39-49.

Yu, D., Ellis, H. M., Lee, E.-C., Jenkins, N. A., \& Copeland, N. G. (2000). An efficient recombination system for chromosome engineering in Escherichia coli. Proceedings of the National Academy of Sciences, 97(11), 5978-5983.

Zha, J., Zang, Y., Mattozzi, M., Plassmeier, J., Gupta, M., Wu, X.,... Koffas, M. A. (2018). Metabolic engineering of Corynebacterium glutamicum for anthocyanin production. Microbial cell factories, 17(1), 143.

Zhang, Y., Buchholz, F., Muyrers, J. P., \& Stewart, A. F. (1998). A new logic for DNA engineering using recombination in Escherichia coli. Nature genetics, 20(2), 123-128.

Zhang, Y., Muyrers, J. P., Testa, G., \& Stewart, A. F. (2000). DNA cloning by homologous recombination in Escherichia coli. Nature biotechnology, 18(12), 1314-1317.

Zhao, N., Li, L., Luo, G., Xie, S., Lin, Y., Han, S.,... Biotechnology. (2020). Multiplex gene editing and large DNA fragment deletion by the CRISPR/Cpf1-RecE/T system in Corynebacterium glutamicum. 1-10.

Zhu, X., Zhao, D., Qiu, H., Fan, F., Man, S., Bi, C., \& Zhang, X. (2017). The CRISPR/Cas9-facilitated multiplex pathway optimization (CFPO) technique and its application to improve the Escherichia coli xylose utilization pathway. Metabolic engineering, 43, 37-45.

This article is protected by copyright. All rights reserved. 


\section{Figure legends}

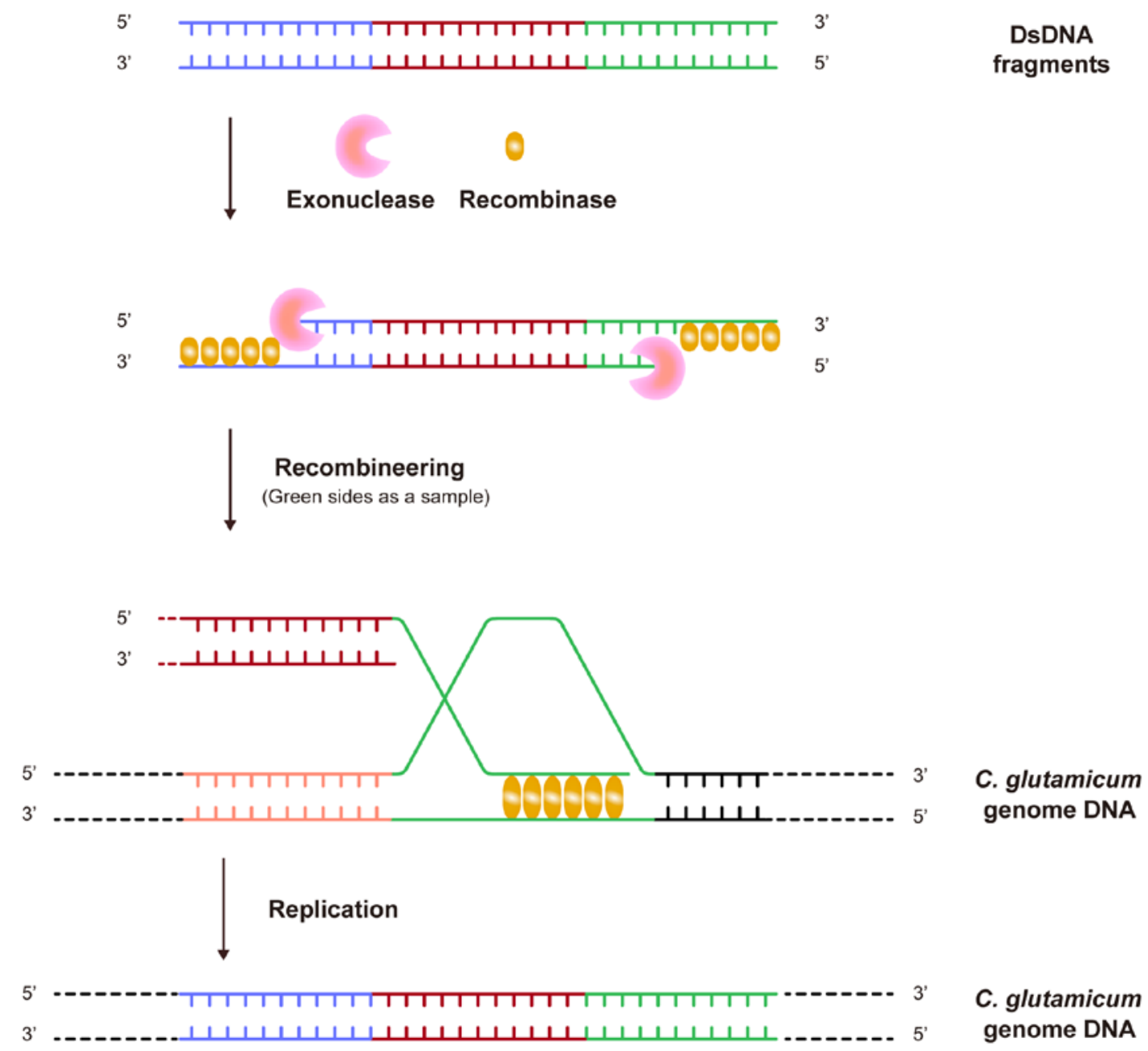

Figure 1. C. glutamicum Recombineering with dsDNA. DNA substrates with homology (blue and green) on either side of a drug-resistance cassette (red) was generated by fusion PCR. The recombinase and exonuclease were expressed with IPTG in C. glutamicum, followed by electoporating the DNA substrates for recombination. The exonuclease digests the genomic DNA strands to generate a 3'-ended ssDNA tail. The recombinase then binds and aligns the transformed DNA with the genomic DNA. After DNA replication, the targeted genomic region (pink) is replaced with the transformed DNA.

This article is protected by copyright. All rights reserved. 
A

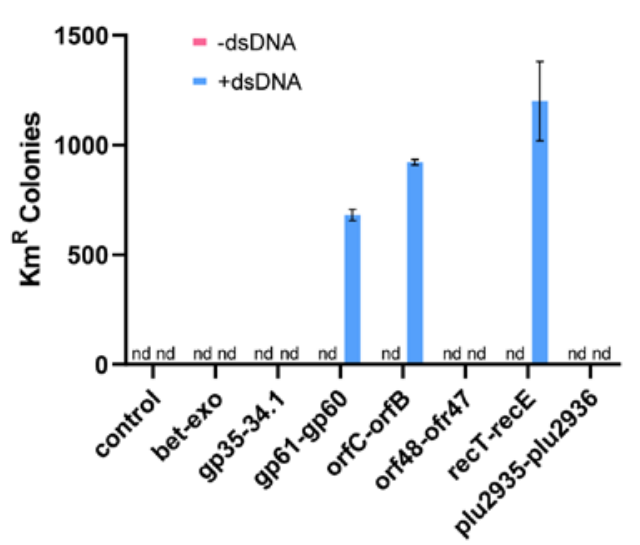

C

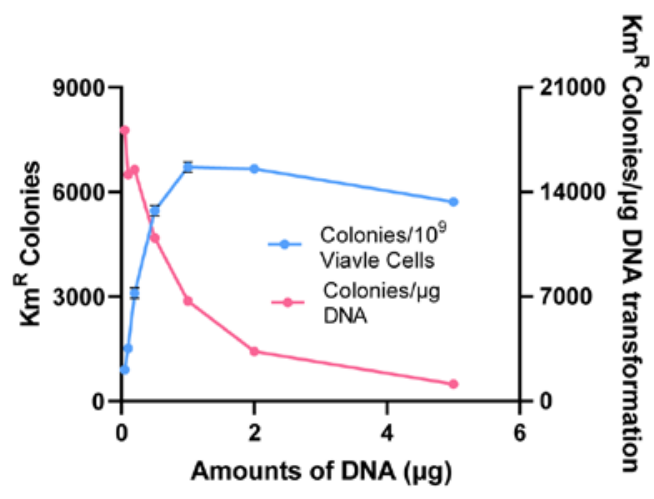

B
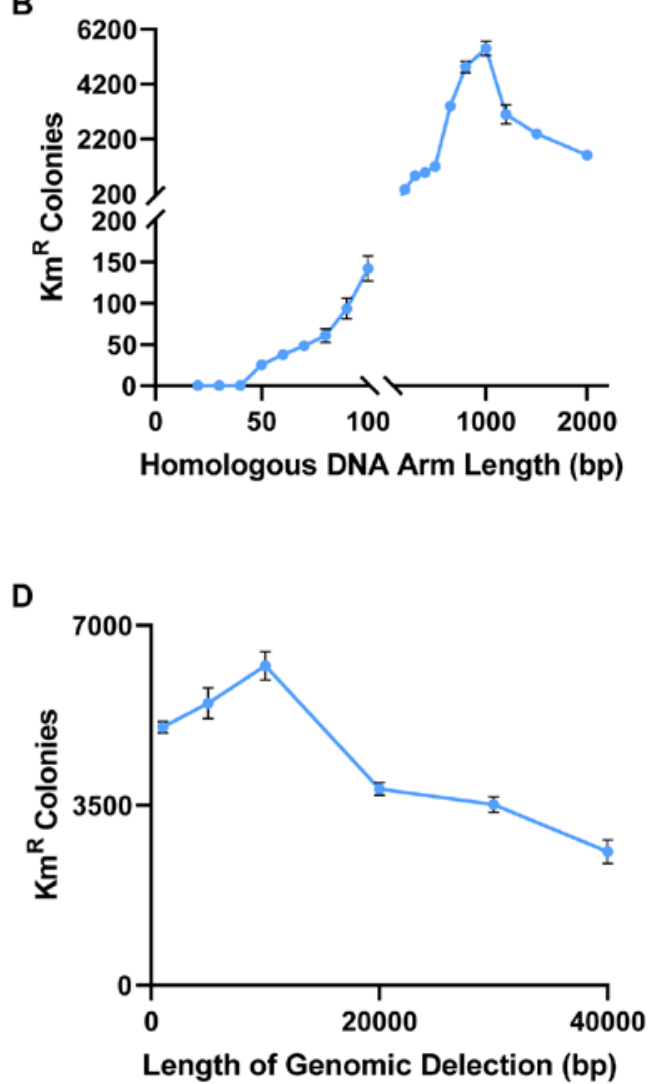

Figure 2. Optimizing dsDNA Recombineering. (A) Three recombinase-exonuclease pairs successfully integrated a kanamycin-resistance cassette into the nonessential gene cgp_2810, represented by the number of kanamycin resistant clones recovered after transformation with 500 ng dsDNA with 400 bp homologous arms. RecET had the highest efficiency with $1200 \mathrm{Km}^{\mathrm{R}}$ colonies $/ 10^{9}$ viable cells. A strain with an empty plasmid pEC_XC99E was used as a control. (B) RecET recombination efficiency is highest with 1,000 bp homologous arms when transforming with 500 ng dsDNA. Efficiencies decreased at homologous arm lengths higher than 1,000 bp. (C) RecET recombination had the highest efficiency with $1 \mu$ g of dsDNA when transforming DNA with 1,000 bp homologous arms (blue). Higher amounts of DNA did not increase transformation This article is protected by copyright. All rights reserved. 
efficiency. As the amount of DNA transformed is increased, the amount of colonies per DNA transformed decreases (red). (D) Using $1 \mu \mathrm{g}$ of dsDNA with 1,000 bp homologous arms, upwards of 40,000 bp could be deleted with one transformation step. All knockouts removed cgp_0721, rendering C. glutamicum colonies white instead of the typical yellow color, allowing for easy screening. The highest efficiency was achieved when deleting a 10,000 bp region.

A

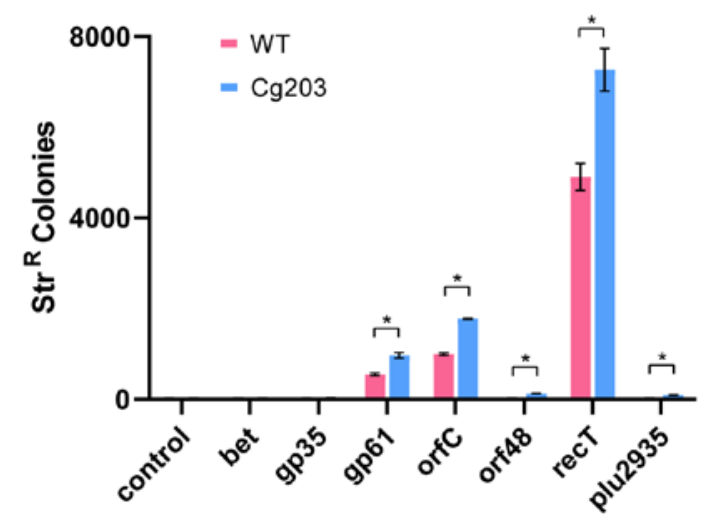

B

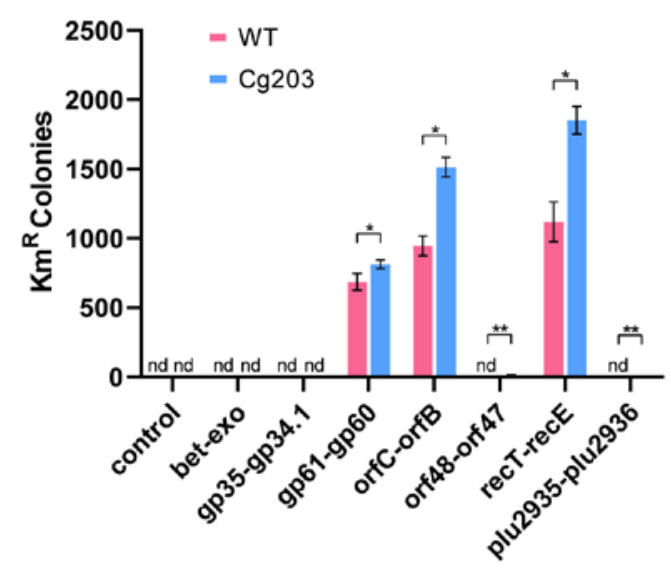

Figure 3. Eliminating Codon-bias Improves Recombination Efficiency. (A)

ssDNA recombineering in the codon-unbiased strain cg203 improved efficiencies for all recombinases except Bet and GP35 when using 500 ng of ssDNA (*, $\mathrm{P}<0.05)$. RecT ssDNA recombination efficiency increased 1.5 fold in cg203 compared to the wild-type. A strain with an empty plasmid pEC_XC99E was used as a control. (B) dsDNA recombineering in the codon-unbiased cg203 improved efficiencies for all recombinase-exonuclease pairs except Bet/Exo and GP35/34.1 when using 400 bp homologous arms and 500 ng of dsDNA (*, $\mathrm{P}<0.05$ ). No colonies were present when using Orf48/47 and plu2935/plu2936 with the wild-type but were present in cg203 (**, P<0.01). RecET dsDNA recombination This article is protected by copyright. All rights reserved. 
increased 1.7 fold in cg203 compared to the wild-type. A strain with an empty plasmid pEC_XC99E was used as a control.
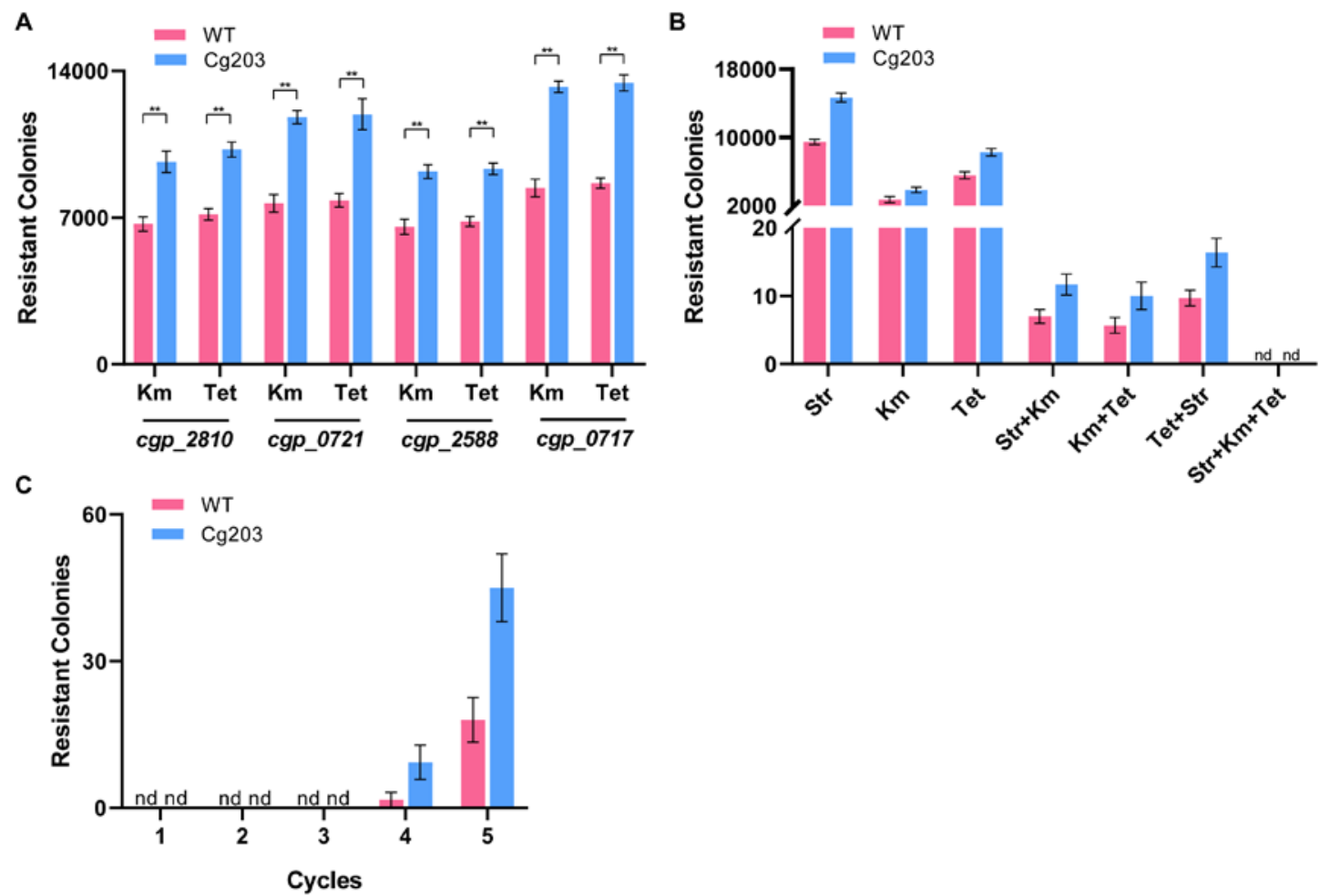

Figure 4. RecET Recombination Can Modify Two and Three Distinct

Genomic Regions. (A) RecET recombineering under optimal conditions ( $1 \mu \mathrm{g}$ dsDNA and 1,000 bp homologous arms) has improved efficiencies in the codon-unbiased strain cg203, for both a kanamycin-resistance cassette and a tetracycline-resistance cassette insert into cgp_2810,cgp_0721,cgp_2588 or cgp_0717 genomic sites (**, $\mathrm{P}<0.01)$. (B) When any two of the kanamycin-resistance, streptomycin-resistance, or tetracycline-resistance cassettes are transformed together, recET recombineering can modify two distinct regions at low efficiencies. Recombineering in Cg203 increases efficiency for the double-modification compared to the WT. No colonies were obtained when all This article is protected by copyright. All rights reserved. 
three cassettes are transformed together. (C) After four cycles of recombineering, recET recombineering can modify three genomic regions at low efficiencies.

Cg203 has a 4-fold increase in efficiency compared to the WT.

Table 1: Comparison of recombinase efficiencies

\begin{tabular}{cccc}
\hline \multirow{2}{*}{ Vector } & \multicolumn{3}{c}{ Str $^{\mathrm{R}}$ per 10 ${ }^{9}$ viable cells } \\
\cline { 2 - 4 } & +oligo $^{\dagger}$ & -oligo & ${ }_{\text {+plasmid }}^{\ddagger}$ \\
\hline pEC-XC99E & 23 & 19 & $2.2 \times 10^{5}$ \\
pEC-bet & 28 & 15 & $2.4 \times 10^{5}$ \\
pEC-gp35 & 31 & 24 & $2.8 \times 10^{5}$ \\
pEC-gp61 & $5.4 \times 10^{2}$ & 18 & $2.4 \times 10^{5}$ \\
pEC-orfC & $9.6 \times 10^{2}$ & 21 & $2.7 \times 10^{5}$ \\
pEC-orf48 & 27 & 26 & $2.4 \times 10^{5}$ \\
pEC-recT & $4.8 \times 10^{3}$ & 19 & $2.6 \times 10^{5}$ \\
pEC-plu2935 & 24 & 21 & $3.2 \times 10^{5}$ \\
\hline
\end{tabular}

"Number of colonies recovered after transformation with 500 ng ssDNA. "500 ng of plasmids carrying rpsL K43R (Supplementary Table 1) was transformed into different strains in the same condition as ssDNA.

This article is protected by copyright. All rights reserved. 